\title{
Réflexions sur la formalisation, en tant que système, d'une transcription des formes des Langues des Signes : l'approche Typannot
}

\author{
Dominique Boutet ${ }^{1 \dagger}$, Claudia S. Bianchini ${ }^{2,{ }^{*}}$, Patrick Doan ${ }^{3}$, Léa Chèvrefils-Desbiolles ${ }^{1}$, \\ Chloé Thomas ${ }^{1}$, Morgane Rébulard ${ }^{3}$, Adrien Contesse ${ }^{3}$, Claire Danet ${ }^{3}$, \\ Jean-François Dauphin ${ }^{3}$, et Mathieu Réguer ${ }^{3}$. \\ ${ }^{1}$ EA7474-DyLIS, Université de Rouen Normandie (France) \\ ${ }^{2}$ UR3816-FoReLLIS, Université de Poitiers (France) \\ ${ }^{3}$ D-sign-E, ESAD Amiens (France)
}

\begin{abstract}
Résumé. Transcrire les langues des signes (LS) nécessite de prendre en considération leur nature gestuelle et de comprendre les raisons (parmi lesquelles centration sur les mains et utilisation d'un cadre de référence égocentré) pour lesquelles des systèmes typographiques (HamNoSys, SignWriting) ont échoué à s'imposer comme outil de transcription. La gestualité met en mouvement tous les segments du membre supérieur selon des degrés de liberté, en fonction d'amplitudes particulières et à travers une série de cadres de référence intrinsèque centrée sur chaque segment. Typannot, le système typographique présenté ici, repose sur ces caractéristiques et les intègre dans une hiérarchie structurelle ménageant un niveau informationnel correspondant aux traits (caractères), rassemblés dans un niveau de glyphes composés. La facilité d'utilisation de Typannot est assurée par le respect de quatre principes de conception (généricité, modularité, lisibilité et inscriptibilité) et l'utilisation d'une interface de saisie ménageant ces niveaux d'informations. Afin d'illustrer l'usage de Typannot, ont été menée des analyses portant sur les configurations et l'emplacement propre de la main, qui montrent l'influence de la gestualité praxique sur la gestualité symbolique (c.-à-d. les signes des LS). Vue la durée d'annotation encore très importante qu'un tel système de transcription impose, l'objectif est de permettre une "dictée gestuelle", une transcription directe à partir des données de capture de mouvement. Cette perspective devrait aussi faciliter la transcription de l'ensemble des gestes dits co-verbaux de n'importe quelle langue vocale.
\end{abstract}

\begin{abstract}
Reflections on the formalization, as a system, of a transcription of Sign Language forms: the Typannot approach. The transcription of sign languages (SL) should take into account the gestural nature of these languages and, above all, why many typefont systems (e.g., HamNoSys, SignWriting) failed in establish themselves as a transcription tool (e.g., given the focus on the hands and the ego-centered frame of reference). Gesturing sets all the segments of the upper limb in motion according to degrees of freedom, particular amplitudes and a series of
\end{abstract}

\footnotetext{
*Contact : claudia.savina.bianchini@univ-poitiers.fr
} 
intrinsic frames of reference centered on each segment. Typannot, the typefont system presented here, is based on these characteristics and integrates them into a structural hierarchy with an informational level corresponding to the features (characters), gathered in a level of glyphs composed. Typannot usability is ensured by respecting four design principles (genericity, modularity, legibility/readability and inscriptibility) and the use of a data entry interfaces managing these information levels. In order to illustrate the use of this font, analyses of the handshapes and proper location of the hand show the influence of practical gestures on symbolic gestures (i.e., SL signs). Considering the still cumbersome annotation time that such a transcription system imposes, the objective is to allow a "gestural dictation", a direct transcription from motion capture data. This perspective should also facilitate the transcription of all the so-called co-verbal gestures of any vocal language.

\section{Introduction}

Depuis les premières tentatives d'éducation des sourds (à la fin du $18^{\mathrm{e}}$ et au $19^{\mathrm{e}}$ siècle) basées sur l'utilisation des Langues des Signes (LS), le problème de la représentation graphique des signes a été posé, donnant lieu à des solutions très variées (cf. Bonnal-Vergès, 2005) : dessins des signes (cf. les dictionnaires des Frères de Saint Gabriel), descriptions en langue vocale (LV) de la forme et du sens des signes (cf. le dictionnaire de l'Abbé Sicard), ou encore ébauche de système d'écriture dédié (cf. la Mimographie de Bébian [1826]). Suite à la reconnaissance des LS comme objet d'étude linguistique (avec le travail fondateur de Stokoe [1960]), le problème de leur représentation à des fins d'étude scientifique a été abordé de façon récurrente (Prillwitz et al., 1989 ; Sutton, 1995 ; Slobin et al., 2001), sans jamais trouver une solution satisfaisant la communauté des linguistes. De nombreux systèmes ont été proposés au cours des 40 dernières années, reflétant l'intérêt des auteurs pour un niveau d'analyse spécifique, leur vision sur la nature langagière des différents éléments caractérisant les LS et sur l'importance des différentes parties du corps pour véhiculer le sens, mais aussi, de manière corrélée, la projection de ce qu'un système de transcription peut et doit représenter. En filigrane de ces réflexions, par-delà la question de la transcription, l'utilité de doter les LS d'un système d'écriture quotidienne revient régulièrement.

Après avoir présenté les principaux systèmes de notation des LS, nous montrerons que les questions soulevées par la transcription concernent tant des gestualités symboliques inhérentes à la multimodalité (gestualité dite co-verbale) que des gestualités plus expressives (danse, marionnettes, cirque). Tenter d'y répondre suppose de modifier le cadre de référence de l'étude des phénomènes gestuels en faisant plus cas du corps et des mouvements qu'il génère. Sur ces bases renouvelées et définies, nous présentons Typannot, un système linguistique et typographique, basé sur une série de polices de caractères, développé par l'équipe GestualScript ${ }^{1}$ pour transcrire les LS : seront identifiés les principes sur lesquels il repose, ainsi que la hiérarchie mise en place (caractères génériques et glyphes composés, cf. infra) permettant tout à la fois de lire et d'écrire aisément les paramètres (configuration de la main, expressions faciales, mouvements, etc.) et d'interroger ces notations sur plusieurs niveaux de hiérarchie (formes et informations constituantes). Une interface graphique de saisie de la notation, Typannot KeyBoard, sera décrite dans ses grandes lignes. Enfin, nous exposerons les résultats d'une étude linguistique portant sur l'inclusion de la gestualité praxique dans les formes de mains et des doigts (configuration) en LS Française (LSF), étude rendue possible par l'utilisation de Typannot. Un manuel de codage exposant et illustrant les définitions de chaque caractère sous le logiciel ELAN est à disposition afin que le public le plus large puisse se saisir de ce système typographique de transcription. 


\section{Représenter les gestualités : la recherche d'un terrain commun}

Différentes classifications des systèmes de représentations des LS sont possibles ${ }^{2}$. Pour la réflexion présentée ici, il est particulièrement important de distinguer entre les systèmes formels de transcription et ceux fonctionnels d'annotation des donnés langagières, mais aussi entre les systèmes représentant l'intégralité du corps du signeurs (doigts et mains, bras et avant-bras, buste et tronc, expressions faciales) et ceux centrés que sur les doigts et les mains.

La représentation fonctionnelle des signes associe à chaque signe une fonction bien précise, indépendamment de sa forme : pointage, prise de rôle, lexème, classificateur, etc. Les étiquettes varient selon le niveau linguistique exploré et la théorie suivie par le chercheur (par exemple, la prise de rôle peut être appelée embodiment ou transfert personnel) mais aussi selon la LV et surtout selon la vision de ce qui est linguistique ou non en LS. En effet, alors que les signes très lexicalisés ont été, dès les débuts des recherches sur les LS, reconnus comme pleinement linguistique, le statut de structures complexes (comme les prises de rôle, par exemple) est encore aujourd'hui sujet de débats (cf. infra, le débat sur la présence d'une gestualité co-verbale au sein des LS).

La représentation formelle des signes, quant à elle, cherche (autant que possible) à fournir une description indépendante des modèles d'analyse fonctionnelle de la langue : il ne s'agit pas de savoir "que fait le signeur" mais "comment est le corps du signeur pour produire les signes". Les tentatives de représenter formellement les signes ont produit une multitude de systèmes (cf. paragraphe suivant), se différenciant par la précision descriptive et la nature plus ou moins motivée des caractères choisis (selon des critères disparates : iconicité, lien avec l'écriture de la LV, etc.). Ces systèmes se distinguent aussi par leur adéquation à être utilisés en milieu scientifique : plus ou moins grande facilité d'apprentissage, de transmission et d'utilisation mais aussi informatisation du système pour accélérer le processus de transcription, pour améliorer l'homogénéité, la transmissibilité, la pérennité et la requêtabilité des données obtenues.

Les problématiques présentées ci-dessus sont partagées par les chercheurs s'intéressant à la gestualité humaine en général (Darwin, 1998 [1872] ; Efron, 1972), qui manquent eux aussi de systèmes pour la représenter : trouver des solutions pour les LS pourrait donc constituer le socle à même de résoudre le problème pour toutes les manifestations gestuelles humaines, qu'elles soient langagières (gestualité co-verbale) ou artistiques (théâtre, cirque, marionnettes, etc.)

\subsection{Les langues des Signes}

Seront présentés ici différents systèmes de représentation formelle des LS afin de montrer que, même s'ils aspirent tous à résoudre le même problème, ces solutions sont toutes très différentes entre elles: Stokoe Notation (phono-sémantique, paramétrique, linéaire, peu iconique et liée à l'alphabet latin) et ses dérivés comme Hamburg Notation System (HamNoSys), SignWriting (SW ; paramétrique, iconique, non-linéaire et non lié à une écriture latine), Berkeley Notation System (BTS ; à la frontière entre système formel et fonctionnel, linéaire, non iconique et très lié à la LV).

(a)

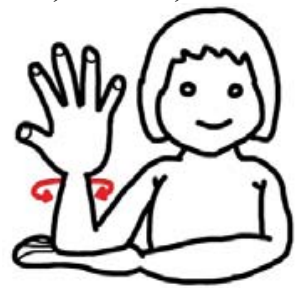

(b)

$$
B<\Lambda^{*} \text { gd 5^> ND } \omega
$$

(c)

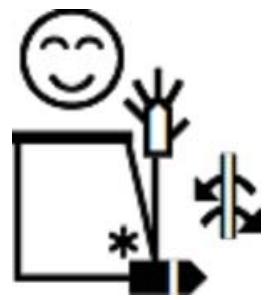

Fig. 1. (a) le signe [ARBRE] en Langue des Signes Française, (b) sa représentation en Stokoe Notation (1960 ; adaptée par Radutzky, 2001) et (c) en SignWriting (Sutton, 1995). 
Le premier système de transcription "moderne" (c.-à-d. post-1960) est Stokoe Notation (Stokoe, 1960). Son but initial étant de répertorier les signes pour réaliser un dictionnaire (Stokoe, 1965), il élabore un système graphique permettant une indexation simple en se basant sur les lettres et les symboles présents sur le clavier d'une machine à écrire, et en proposant de les disposer linéairement. De plus, bien que conscient de la limite de cette approche (Stokoe, 1960), il décide de ne représenter que les composantes manuelles des signes. Il réalise ainsi une décomposition paramétrique des LS qui concilie la phonologie et la sémantique. Stokoe distingue 4 paramètres manuels - la configuration, l'emplacement, le mouvement et l'orientation ${ }^{3}$ - qui doivent tous nécessairement apparaître afin de pouvoir réaliser (et donc représenter) un signe, unité minimale de sens reconnue par l'auteur.

Ainsi, en Stokoe Notation, le signe [ARBRE] en LSF (cf. Fig. 1) est représenté par la séquence ' $\mathrm{B}<\Lambda^{*} \operatorname{gd} 5 \Lambda>\mathrm{ND} \omega$. ${ }^{4} 5$. En première approche, cette représentation ne semble pas liée à la langue (vocale) dominante. Pourtant, ce n'est pas le cas : le choix d'identifier une configuration 'main plate doigts serrés' par 'B' est lié à la façon dont, en LS américaine (et en LSF), on représente la lettre B en dactylologie (l'alphabet manuel). Or, les LS varient d'un pays à l'autre, leur représentation de l'alphabet peuvent changer également (par exemple 'A' en LSF se réalise comme ' $S$ ' en LS italienne, et vice-versa), liant ainsi la compréhensibilité de Stokoe Notation à son adaptation à la LS qu'elle doit coder.

Selon Stokoe (1960; mais aussi, plus récemment, selon Sandler, 1989 ; Johnson \& Liddell, 2010), le choix au sein de chaque paramètre répond à un fonctionnement phonologique qui se double d'une motivation iconique en rapport avec la signification véhiculée par le signe. Dans le signe [ARBRE] l'avant-bras et la main dominants figurent un tronc d'arbre surmonté d'une ramure (doigts écartés) ; tandis que la main dominée (plate et horizontale) figure le sol sur lequel repose le tronc. Chaque paramètre véhicule donc une information de type iconique, et la globalité du signe signifie 'un arbre'.

Depuis sa création, Stokoe Notation a été prise comme modèle par de nombreux autres systèmes de notation (cf. note 4) parmi lesquels HamNoSys (Prillwitz et al., 1989). Suivant les mêmes principes descriptifs que son prédécesseur (subdivision en paramètre, description linéaire, etc.), HamNoSys présente de nombreuses améliorations par rapport à son modèle : dépassant la limite des touches présentes sur le clavier, les créateurs de HamNoSys ont essayé d'accroître l'iconicité (et donc la lisibilité) de leurs symboles. Même marginalement, la description de quelques composantes non manuelles est incluse. Requêtable par ordinateur, HamNoSys est facilement scriptible par les logiciels communs d'annotation (ELAN ou ANVIL), voire dans un logiciel de base de données spécialement conçu (iLEX). Toutefois la lenteur de la transcription et la difficulté de faire des requêtes spécifiques d'éléments au sein de caractères contenant plusieurs informations posent problème: chaque symbole représentant une configuration montre la forme de toute la main mais ne permet pas de rechercher la forme de chaque doigt.

Suivant le même type de description paramétrique mise en place par Stokoe, d'autres chercheurs ont proposé de représenter les LS sans recourir à des symboles. Ainsi, différentes réalisations paramétriques sont décrites en $\mathrm{LV}$ : aux classiques ' 5 ' ou ' $\mathrm{B}$ ' issus de la dactylologie et de la numération en LS sont substitués des 'clef', 'bec de canard', '2 jambes' 'OK'. C'est le cas notamment du BTS (Slobin et al., 2001), système linéaire décrivant les paramètres en leur associant une description par mot qui laisse transparaître plus le signifié que le signifiant d'un signe. Si ces descriptions sont facilement encodables et requêtables sur ordinateur, elles rendent difficiles les recherches centrées sur la forme : il est par exemple impossible de savoir que 'o' et 'bec de canard' sont deux configurations ne se distinguant que par la forme des doigts, recourbés dans le premier cas, droits dans le deuxième.

En marge de la multitude de systèmes linéaires plus ou moins dérivés de Stokoe Notation, on trouve SW (Sutton, 1995 ; pour une analyse cf. Bianchini, 2012). Inventé par une chorégraphe et utilisé surtout en milieu pédagogique, il est l'adaptation aux LS d'un système plus vaste (le Sutton Movement Writing ; Sutton, 1972) qui se propose de représenter toute 
la gestualité humaine ; au-delà d'un simple instrument de transcription, il est présenté comme un système d'écriture quotidienne des LS. SW se compose de symboles hautement iconiques agencés dans un espace bidimensionnel (et non plus linéaire) qui calque la réalité corporelle du signeur, ce qui lui assure une très grande lisibilité, récapitulant en une image un signe, un peu à la manière d'un dessin instantané ou d'une photographie. Des personnes ne maîtrisant peu ou pas ce système peuvent le déchiffrer à défaut d'en être lecteur. De plus, SW est totalement indépendant de toute LV et de ses systèmes d'écriture. Le signe est toujours subdivisé de façon paramétrique et phonologique avec ses composantes manuelles autant que non-manuelles (expressions faciales, regards mais aussi mouvements du buste et de la tête). Notons toutefois que SW n'est pas un instrument conçu pour la recherche linguistique : il est difficilement requêtable et impossible à utiliser dans des logiciels d'annotations (Bianchini, 2012). De plus, sa grande lisibilité ne s'accompagne pas d'une facilité scripturale équivalente, surtout dans sa version informatisée. Sa chance d'être adapté (plus qu'adopté) par les linguistes pour satisfaire leurs besoins de représentation des LS reste faible, sans toutefois que ceci nuise à son attractivité pour la communauté sourde.

La sélection de systèmes présentée ici montre que, face aux défis de la représentation des LS, les réponses données sont multiples mais aucune n'arrive vraiment à satisfaire l'ensemble des exigences posées par la communauté scientifique (besoin de données fiables, lisibles, scriptibles et requêtables). L'absence d'un système efficace amène tout naturellement à une pénurie criante de grands corpus transcrits et, partant, à la quasi-impossibilité de mettre en commun les ressources linguistiques transcrites au sein des différents laboratoires qui, souvent, utilisent des méthodes différentes et parfois incompatibles.

\subsection{Les gestualités entendantes : gestes co-verbaux et artistiques}

Depuis la fin des années '90, la question de la part gestuelle dans les LS est débattue (Singleton et al., 1995 ; Liddell, 2003 ; Schembri et al., 2005 ; Goldin-Meadow \& Brentari, 2017). Les arguments en faveur d'une séparation entre signes et gestes co-verbaux convergent tous vers la nécessité d'une meilleure compréhension du paramètre du mouvement. Selon Goldin-Meadow \& Brentari (2017), tant que le mouvement ne sera pas bien décrit et compris, ce débat sur la présence ou l'absence de gestes co-verbaux dans les LS ne sera pas tranché. Le renvoi vers la gestuelle co-verbale de certain(e)s (parties de) signes est toujours fait dans une approche négative et de manière systématique à partir des fonctionnalités de ce qui relèverait du langage pour les signes ; le précipité final étant dévolu à la catégorie des gestes non/co/verbaux. A aucun moment ces supposés gestes co-verbaux présents dans les LS ne sont mis en regard de manière formelle avec les formes des gestes co-verbaux qui accompagnent les LV. L'absence de système de transcription commun entre les LS et la gestualité des phénomènes multimodaux des LV, de ce point de vue, revêt une urgence particulière; spécialement si on veut comparer les deux types de gestualité co-verbale sur une base formelle commune et non pas sur des fonctionnalités langagières dépendantes et peut-être redevables des modalités voco-acoustique d'une part (LV) et visiogestuelle (LS) d'autre part. L'enjeu relève des niveaux d'attribution langagière des phénomènes gestuels : se définissent-ils par leur fonction seulement ou bien peut-on aussi prendre en considération leur forme?

Ces questions de ressemblance formelle ne concernent pas seulement la gestuelle symbolique en lien avec le langage, elles touchent également des gestualités expressives voire artistiques. Un des modèles linguistiques le plus reconnu de structuration du fait gestuel (le continuum dit de Kendon [McNeill, $1992 ; 2005]$ ) réserve une place à la pantomime sans que celle-ci ait peut-être à voir avec la pantomime artistique (Boutet et al., 2010). De nombreuses études sur les LS recourent également à la pantomime comme une sorte de déversoir de ce qui ne serait pas proprement langagier dans ces productions signées (Bellugi \& Klima, 1979 ; Emmorey, 2003 ; Emmorey et al., 2011 ; Sandler, 2013 ; Abramova, 2018). 
Ici encore l'impérieuse nécessité d'une notation commune apparaît, cette fois entre des phénomènes langagiers et artistiques. Les notations de phénomènes gestuels symboliques comme la danse sont nombreux (Benesh \& Benesh, 1956; Hall, 1967 ; von Laban \& Lange, 1975 ) et anciens pour certains (Tabourot, 1588; Feuillet, 1701), mais aucun système de notation de la pantomime n'existe malgré une scène riche et des propositions fortes et variées, en particulier en France (le mime Marcel Marceau [Kühnl-Kinel, 1999] ; Lecoq et al., 1997 ; Decroux \& Pezin, 2003). L'Institut International de la Marionnette (IIM) dans une chaire commune avec le cirque (Chaire d'Innovation Cirque et Marionnette, CiMa) tente depuis 2016 d'adapter les notations de Benesh et de Laban aux exigences circassiennes et à celles de la marionnettique. Si le paysage est contrasté en matière de notation pour les arts vivants, il ressemble à ce qui existe pour les notations de gestualités symboliques langagières. Les mêmes nécessités patrimoniales (sauvegarde, conservation, base pour des re-créations), de lisibilité/inscriptibilité (précision, distinctivité, reproductibilité), d'analyse (décomposition des techniques et des courants, comparaison des systèmes symboliques ou expressifs, voire mise au jour de grammaire) et d'enseignement (gamme d'exercices, prise de notes, support d'apprentissage) poussent évidemment à unifier ces notations de gestes symboliques langagiers et artistiques sous une bannière commune, telle qu'a connu l'Europe dans l'adoption d'un alphabet latin écrit ou plus récemment à une plus grande échelle, un API. Sans préjuger de particularités selon les arts ou les expressions symboliques (gestes co-verbaux ou LS), cette base de notation partagée devrait offrir un canon commun permettant des comparaisons formelles entre les LS, ainsi qu'entre systèmes symboliques jugés comme distincts voire différents par nature.

\section{La proposition graphématique}

\subsection{Cadre de référence}

Nous avons vu (cf. §1) que les notations existantes pour la partie manuelle des LS font grand cas des mains et des doigts et très peu des autres segments (épaule, bras et avant-bras). Tout se passe comme si une approche implicite plaquait le sens gestuel sur les articulateurs les plus distaux - main et doigts - et laissait aux autres segments le soin de porter les mains et de les déplacer le cas échéant (signes à trajectoire, cf. Brentari, 1998:4,136) sans interroger les relations entre les segments, ni d'ailleurs leur rôle dans la construction du sens. Il semble qu'une conception du sens fondée pour l'essentiel sur la modalité de réception prévale autant pour les LV (audition) que pour les LS et la gestualité (vision). Dans cette manière d'étudier les LS, la production du sens tend à se détacher du support qui réalise les formes, c'est-à-dire du corps même, pour ne plus saisir que l'ultime limite de son extériorisation (les mains et les doigts) ou la surface du visage (mimique, gestes de bouche), à l'instar de la voix dont le signal est détaché des moyens de production et de sa structuration (les gestes du tractus phonatoire). Certes, l'étude d'un objet est facilitée par l'usage de moyens utilisés pour l'analyse d'un autre objet dont la connaissance est majeure (ici les LV) mais cette économie d'effort peut-elle se faire, pour la gestualité et les LS, au prix d'une désincarnation ?

D'une manière similaire, le cadre de référence adopté pour l'étude des gestes et des LS est un cadre de référence relatif égocentré, celui majoritaire dans les langues indo-européennes (pour la notion de cadre de référence cf. Talmy, 1983 ; Levinson, 1996 ; pour la prédominance de l'égo-centration dans les langues indo-européennes cf. Brown \& Levinson, 1993 ; Pederson et al., 1998). Malgré le fait que de nombreuses études aient montré qu'il ne s'agissait pas d'un cadre de référence universel (Dokic \& Pacherie, 2006), la gauche et la droite, le haut, le bas, l'avant-arrière constituent les directions, les positions et les localisations par lesquelles les signes et les gestes sont analysés. Ce qui peut être compris 
pour des segments qui sont déplacés dans l'espace (les mains et les doigts attachés), l'est plus difficilement pour les épaules qui restent tout de même collées au buste.

Actuellement les paramètres manuels des LS présentent deux canons de mesure, en dehors des configurations : la position pour laquelle on a les paramètres de l'emplacement et du mouvement, et l'orientation de la paume précisément pour le paramètre de l'orientation. L'orientation comme mesure oblige à considérer non pas un point mais un plan, celui de la paume. Enfin la configuration ajoute à la complexité de ce qui est mesuré puisqu'il s'agit d'une forme. Ainsi actuellement, dans la part manuelle de l'analyse des LS, on a un cadre de référence unique - égocentré - pour trois canons de mesure - position, plan et forme - pour finalement un seul segment mesuré (la main comprenant les doigts). On est donc en face d'une limitation drastique des éléments mesurés (la main), avec une grande hétérogénéité des moyens de mesure - de la position à la forme - dans un seul cadre de référence.

Or, un autre cadre de référence plus proche des possibilités de mouvement de chaque segment peut être utilisé pour l'analyse : un ou plutôt des cadres de référence intrinsèques dépendant de chacun des segments (épaule, bras, avant-bras, main). Ce type de cadre - intrinsèque - présente l'avantage : i/ d'offrir un seul canon de mesure, c'est-à-dire la position ; ii/ d'être au plus proche des possibilités de mouvement de chaque segment; et iii/ de pouvoir prendre la mesure de l'ensemble des segments du membre supérieur.

\subsection{Géométrie des degrés de liberté (DDL)}

Que mesurer sur chaque segment dans autant de cadre de référence intrinsèque lorsque seule la position est considérée ? Les degrés de liberté (DDL), qui répondent à la rotation d'un segment autour d'un axe par rapport à un autre. Le mouvement de la main est apprécié par rapport à la position de l'avant-bras, celui de l'avant-bras par rapport au bras et ainsi de suite en remontant jusqu'à l'épaule. Généralement l'axe d'un DDL passe à travers une articulation, mais ce n'est pas toujours le cas ${ }^{6}$. Chaque DDL est donc défini selon un axe de rotation et une amplitude (exprimée en degrés) couverte par le mouvement qu'il rend possible.

(a)

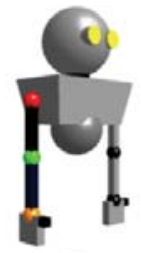

(b)

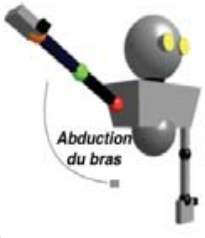

(c)

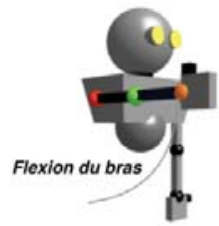

(d)

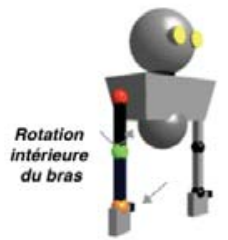

Fig. 2. Degrés de liberté du bras, (a) à partir d'une position de base, (b) ABD, (c) FLEX et (d) RINT.

Pour le bras, on distingue trois DDL: i/ l'abduction/adduction (ABD/ADD ; mouvement dans le plan frontal, cf. Fig. 2b) ; ii/ l'extension/flexion (EXT/FLX) qui porte le bras dans un plan para-sagittal en arrière et en avant d'un plan frontal (cf. Fig. 2c); iii/ un mouvement de rotation extérieure/intérieure (REXT/RINT)pour lequel l'humérus tourne sur lui-même, mouvement rendu visible ici au niveau de la main (cf. Fig. 2d).

(a)

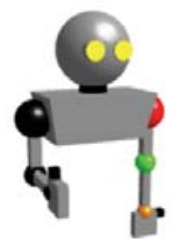

(b)

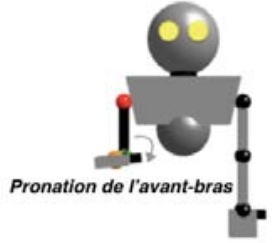

(c)

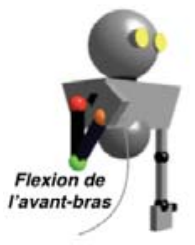

Fig. 3. Degrés de liberté de l'avant-bras, (a) à partir d'une position de base, (b) PrONO (c) FLEX.
L'avant-bras présente deux DDL: i/ la prono-supination (PRONO/SuPI), mouvement de rotation des deux os de l'avant-bras l'un sur l'autre (cubitus et radius) dont la conséquence 
se voit sur la main, pour la SuPI le pouce est dirigé vers l'extérieur, tandis que pour la PRONO le pouce est dirigé vers l'intérieur (cf. Fig. 3b); ii/l'extension/flexion (EXT/FLX) selon laquelle l'avant-bras s'aligne avec le bras (EXT) ou se rapproche de lui (FLX ; cf. Fig. 3c).

(a)

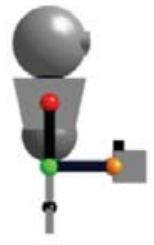

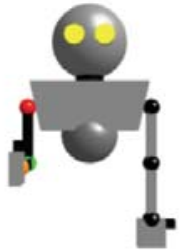

(b)

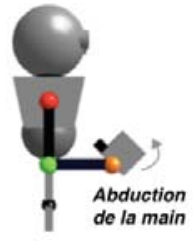

(c)

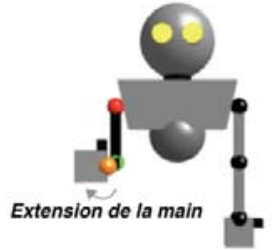

)

Fig. 4. Degrés de liberté de la main, (a) à partir d'une position de base, (b) ABD et (c) EXT.

La main a deux DDL: i/l'adduction/abduction (ADD/ABD) porte la paume vers l'auriculaire ou vers le pouce (cf. Fig. 4b) ; ii/ l'extension/flexion (EXT/FLX) amène la main vers le dos de l'avant-bras ou vers sa partie charnue (cf. Fig. 4c).

Les DdL présentés ici de façon séparée, dont les amplitudes ne sont pas équivalentes, se combinent entre eux dans la réalisation des signes et des gestes. Les pôles de chaque degré (SuPI vs. PRONO ou bien ABD vs ADD par exemple) peuvent être porteurs de sens pour les signes comme pour la gestualité. Ainsi Boutet a montré, pour plusieurs LS et pour la gestualité co-verbale (française) que les pôles de PRONO et d'ADD de la main sont systématiquement impliqués dans la production d'assertions négatives (Boutet, 2015) et que, pour étudier l'expression gestuelle du temps (gestualité comme LS), il faut coupler les cadres de référence égocentré et intrinsèques (Boutet, 2018).

\section{La proposition typographique}

\subsection{Typannot, un système typographique de transcription des LS}

Pour les différentes raisons que nous venons d'exposer, la transcription des LS nécessite la création d'un système capable de représenter et d'articuler les différents niveaux de description des composantes des gestes et des signes. Dans cette partie, nous allons présenter les principes de fonctionnement du système de transcription Typannot qui, pour chaque paramètre, repose sur une décomposition, une organisation et une représentation systématique des caractéristiques corporelles et articulatoires des LS.

\subsection{Structuration du signe et de l'information}

Un système de transcription a besoin de pouvoir représenter les caractéristiques informationnelles de manière distincte et structurée: dans le cas de Typannot, cette organisation mènera à la création d'une hiérarchie de paramètres, parties, variables et valeurs ${ }^{7}$. Un signe de LS est composé de plusieurs paramètres qui constituent un $1^{\text {er }}$ niveau d'information, les paramètres. Chaque paramètre est composé d'un certain nombre d'éléments distincts, les parties, qui constituent le deuxième niveau d'information. Par exemple pour le paramètre "configuration de la main droite" les parties seront les cinq doigts, qui peuvent avoir des positions (sujettes aux différents DDL) ou des interactions entre eux (les valeurs) : les valeurs de notre système seront le fait qu'un doigt peut être courbé ou plat (valeurs de la variable forme), avoir des angles d'ouverture/fermeture différents (valeurs de la variable angle), ou encore être en relation avec un autre doigt (valeurs de la variable interaction). La description de ces quatre niveaux d'information permet de représenter phonologiquement les signes de LS depuis un cadre de référence intrinsèque, celui correspondant à l'organisation corporelle du signeur. La granularité relativement forte qui en 
découle nous permet d'adresser, d'une part, la complexité des combinatoires possibles, et d'autre part, la variété des modes d'expression d'un même signe. Elle s'inscrit ainsi dans une approche praxématique de l'étude de la langue, où les régularités signifiantes sont induites par une analyse bottom-up de corpus larges et variés.

\subsection{Principe de conception}

Notre démarche de design résulte de la prise en compte des problématiques linguistiques et techniques liées à la transcription et ses outils (logiciel, matériel informatique). Elle peut se résumer à quatre principes de conception : généricité, lisibilité, modularité et inscriptibilité ${ }^{8}$.

La généricité correspond au fait de discrétiser l'ensemble des composantes morphologiques (niveaux des traits en phonologie) et gestuelles qui structurent les LS. Au niveau typographique, cette décomposition systématique aboutit à un répertoire limité de symboles dont les multiples combinaisons permettent de représenter un très grand nombre de signes de LS. Grâce à une syntaxe qui reprend les trois derniers niveaux de description (parties, variables et valeurs), le transcripteur peut produire, manipuler et partager une transcription qui, sous sa forme "générique", est hautement décomposée et parfaitement requêtable. Il sera alors possible de rechercher non seulement un paramètre dans sa globalité (la main) mais aussi une de ces parties (l'index) ou encore une de ses valeurs (le doigt recourbé). Ceci représente l'une des plus importantes valeurs ajoutées de Typannot par rapport aux autres systèmes de notation développés jusqu'à aujourd'hui (cf. §1).

Tableau 1. Caractères génériques permettant la décomposition du paramètre configuration de la main.

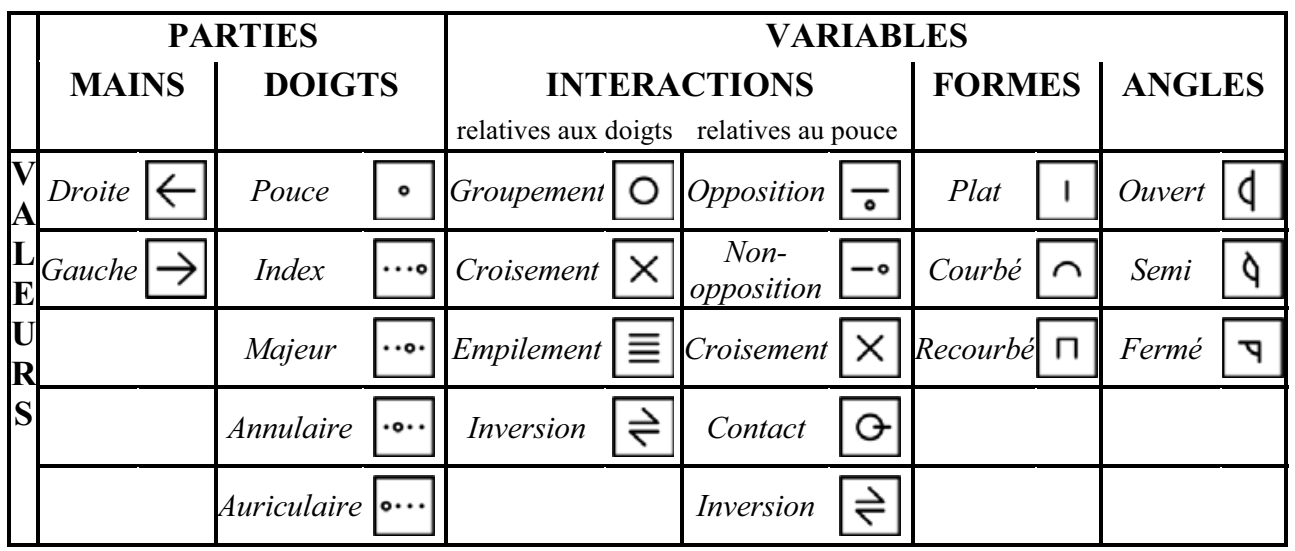

La lisibilité est un concept qui renvoie à la facilité de lecture que confère une certaine forme d'écriture (McMonnies, 1999). Ce critère pose la question du type de lecture permis par la forme que prend la transcription. Alors que la forme "générique" privilégie une lecture analytique et fondamentalement décomposée, le concept de lisibilité que nous introduisons cherche à satisfaire le besoin de lire globalement une transcription comme si celle-ci correspondait à une instance paramétrique connu, immédiatement "prononçable" par la main. Nous pensons que toute transcription des LS peut renvoyer à un savoir implicite, un cadre de représentation intuitivement partagé par l'ensemble des scripteurs et lecteurs : le corps. Ainsi, au côté du mode de représentation phonologique "générique", Typannot propose un mode de représentation logographique du signe de LS qui fusionne les données discrétisées en un glyphe dit "composé" (cf. Fig. 5). Ce dernier est un symbole typographique qui rassemble les traits saillants permettant de figurer par analogie les différentes dimensions et caractéristiques de la main, du visage, des bras, etc. Grâce aux fonctionnalités avancées du format de fonte OpenType (notamment la gestion de ligatures complexes), il est possible de basculer d'un mode d'affichage à l'autre sans perdre les données intrinsèques de la transcription. 
Le registre de glyphes composés nécessite cependant que nous puissions produire systématiquement l'ensemble des combinatoires possibles au sein de chaque paramètre, ce qui correspond à plusieurs centaines de milliers de combinaison. A partir de l'étude d'Eccarius \& Brentari (2008), un petit nombre de modules graphiques (moins d'un millier) a été désigné pour pouvoir s'assembler correctement et former un symbole lisible et cohérent, quelle que soit la configuration phonologique. Grâce à la modularité du bâti glyphique et à un travail de programmation, la construction des glyphes composés est un processus entièrement automatisé.

(a)

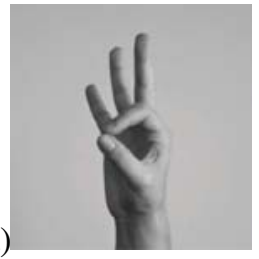

(b)

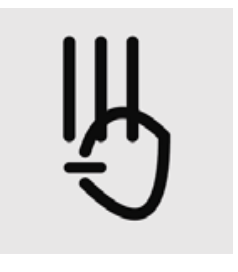

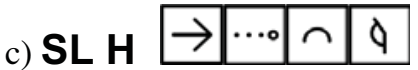

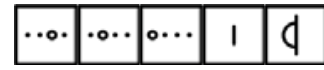

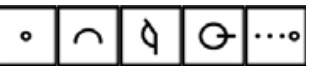

Fig. 5. Glyphe composé (b) et caractères génériques (c) correspondant à une configuration de la main (a) ; les caractères génériques (c), disposés selon une syntaxe fixe, sont la décomposition du glyphe composé (b).

Enfin, le concept de inscriptibilité renvoie à la capacité du scripteur à s'inscrire dans les différentes dimensions de représentation que permet le système de transcription. La modalité visio-gestuelle qu'utilisent les LS nous permet d'aborder l'écriture non pas selon une rupture sémiotique conventionnelle (propre aux écritures - qui sont visuelles - codant la parole), mais en adoptant un mode de représentation littérale des composantes du corps-signe. Rappelons que pour les LS, l'oral comme l'écrit partagent la modalité visio-gestuelle. A priori, l'écrit ne se limite donc pas à une simple inscription de ce que l'oral produit, comme pour les LV, mais, pour les LS, il s'agit de pousser le locuteur à s'inscrire (le réfléchi renvoyant au corps propre) autant dans l'oral qu'il produit que dans l'écrit. La perspective intrinsèque de la transcription phonologique se matérialise au travers des dispositifs graphiques (glyphes composés) et informatiques (interfaces de saisie) permettant au transcripteur de retrouver les rapports sémiotiques qui sous-tendent son activité de scripteur/signeur.

Le fonctionnement de Typannot a été exemplifiée à travers le paramètre de la configuration de la main; toutefois, il est important de préciser que les formules graphématiques et les caractères génériques permettant de représenter la localisation initiale, le mouvement et les paramètres non-manuels sont d'ores et déjà opérationnels ${ }^{9}$.

\subsection{Interfaces de saisie}

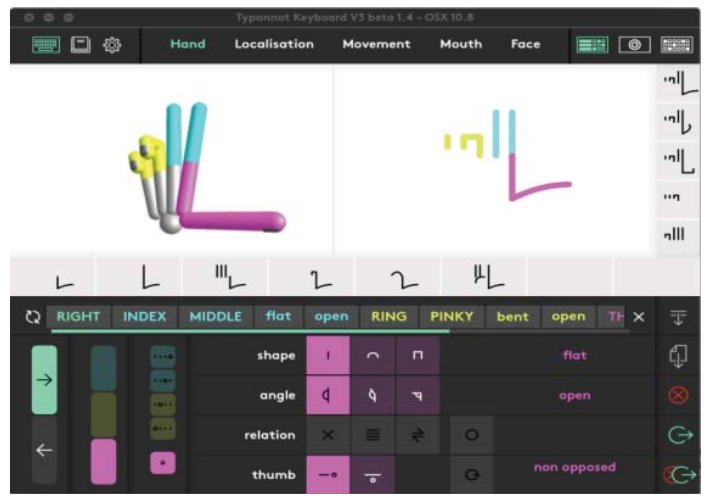

Fig. 6. Interface graphique paramétrique servant à décrire la configuration de la main. 
Les dispositifs de saisie traditionnels, tel que le clavier d'ordinateur, ne sont pas adaptés à ce type de système de transcription. Typannot propose plusieurs interfaces graphiques qui permettent à l'annotateur de décrire simplement chaque composante du signe LS en interagissant directement avec des niveaux de représentations correspondant et qui s'explicitent mutuellement (cf. Fig. 6). Par exemple, un modèle 3D d'une certaine configuration de la main correspond à une séquence de caractères génériques et à un glyphe composé. Parallèlement à celles-ci, grâce au développement des technologies de reconnaissance du corps, des interfaces de captation par le geste sont étudiées.

\section{Résultats préliminaires et analyses}

Les résultats et analyses présentés ci-dessous sont issus d'annotations faites avec Typannot: ils n'auraient pu advenir sans cette police de caractères.

L'un des projets mené avec Typannot pose l'hypothèse que la gestuelle praxique - les gestes quotidiens et incessants de manipulation d'objets - influence la gestuelle symbolique des productions en LS. Cette hypothèse se fonde sur le fait que l'équipement manuel des LS, à l'inverse de l'équipement phonatoire dont l'usage est exclusivement langagier, porte une double fonction : il sert à la fois à la communication orale et à l'interaction avec le monde.

Pour tester la validité de cette hypothèse, nous avons enregistré une locutrice sourde signante en lui soumettant des inputs couvrant différents genres littéraires (narratif, descriptif, procédural, explicatif). L'analyse de ce corpus, menée sur ELAN (Sloetjes \& Wittenburg, 2008) est basée sur l'étude de la différenciation des saisies de force ([i] pouce non opposé aux autres doigts, [ii] pesée de l'objet saisi sur le poignet par une position d'ADD de la main) et des saisies de précision ([contra-i] opposition du pouce face aux autres doigts, [contra-ii] légèreté de l'objet saisi entraînant une position d'EXT du poignet) proposée par Napier (1956). Une transcription détaillée des configurations manuelles, de la localisation initiale ainsi que du mouvement a été réalisée sur 471 signes. La généricité de Typannot nous a permis de faire des requêtes à différents niveaux de manière isolée (configuration) ou en combinant plusieurs paramètres (configuration, localisation et mouvement). Les résultats sont encourageants et, comme dit, n'auraient pas pu voir le jour sans une transcription Typannot : les signes ayant un pouce opposé en contact avec l'un des doigts [contra-i] sont majoritairement en EXT [contra-ii] (63,24\% en EXT contre 14,71\% en FLX de la main), ce qui correspond aux caractéristiques de la saisie de précision. Les signes présentant des configurations avec un pouce non opposé [i] opèrent en grande partie un mouvement d'ADD de la main [ii] (49,45\% en ADD contre 7,69\% en ABD).

Un bon accord inter-annotateur certifie la robustesse de cet ensemble de résultats (corrélation de 0,75 ) et permet de poser les premiers jalons d'une étude des signes non plus basée exclusivement sur une iconicité d'image, mais sur une iconicité corporelle, dont l'influence agit à un niveau de structuration profond des LS, à un niveau phonologique.

Le second corpus étudié et analysé avec Typannot est composé de quatre récits de fictions interprétés par Philippe L'Huillier et Khadra Abdelgheffar, issu du projet LS-Colin ${ }^{10}$.

Pour ce corpus, l'intégralité des paramètres manuels, la configuration ainsi que la localisation initiale et le mouvement sont annotés pour l'ensemble des 634 signes.

Contrairement au postulat qui présuppose que chaque paramètre fonctionne indépendamment l'un de l'autre (né à la suite des travaux de Stokoe [1960]), il est ici question de les appréhender selon leurs liens et leurs possibles influences (Boutet \& Garcia, 2007). Plutôt que de présenter des résultats encore trop parcellaires, nous voudrions souligner le fait qu'une telle étude réclame un temps long de transcription. Le paradoxe est bien là : si ce type d'études ne peut être mené sans une transcription formelle précise situant les signes dans des cadres de référence intrinsèques centrés sur chaque segment du membre supérieur, en l'état actuel, elle nécessite une phase conséquente de transcription. 


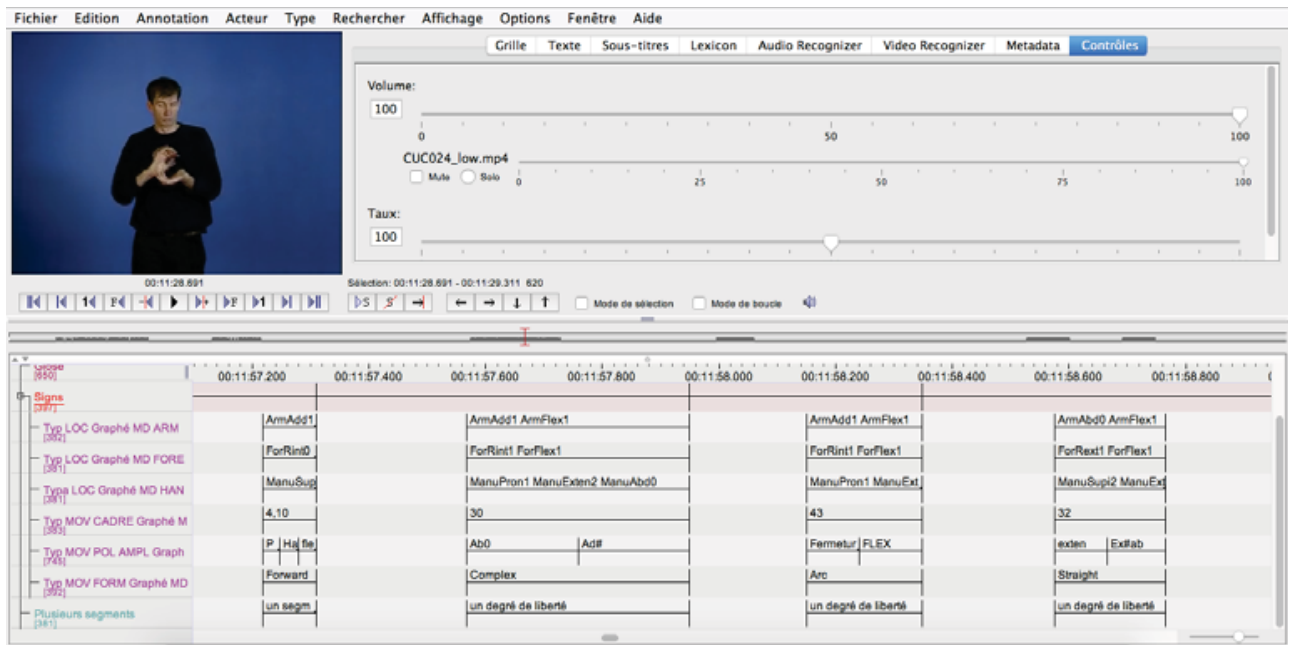

Fig. 7. Extrait du corpus LS-COLIN transcrit avec la police de caractères Typannot.

Comme nous venons de le voir, Typannot permet de noter un niveau formel extrêmement fin. Pour une minute de corpus annoté, il aura fallu $9 \mathrm{~h} 35$ d'annotation (plus de $3 \mathrm{~h}$ pour la configuration, $2 \mathrm{~h} 40$ pour la localisation et $3 \mathrm{~h} 45$ pour le mouvement). Tout paramètre confondu, les 8 min constituant ce corpus ont demandé environ $77 \mathrm{~h}$ de travail.

Ce lourd ratio n'est pas propre au système Typannot: tout travail sur corpus de LS demande une granularité de transcription conséquente et coûteuse en temps. Chételat-Pelé (2010), ayant étudié les paramètres non-manuels d'un corpus de LSF, spécifie que la première phase de sa transcription a duré $15 \mathrm{~h}$ pour une minute de vidéo (1/54000). Boutet \& Blondel (2016) mentionnent un travail nécessitant, quant à eux, $90 \mathrm{~h}$ de transcription pour $1 \mathrm{~min} 27 \mathrm{sec}$ de corpus sur la gestuelle co-verbale.

Afin de faciliter la prise en main de l'outil de transcription Typannot nous avons consigné les différentes modalités de transcription dans un manuel de codage (Bianchini et al., 2018 ; déposé sur le site du Consortium CORLI). Ce manuel est évolutif et sera actualisé à mesure des améliorations du système de transcription.

\section{Conclusion}

Les durées de transcription sont accrues avec Typannot, au moins dans un premier temps. La contrepartie réside dans la précision qu'un tel système de transcription apporte. Il reste à améliorer ce ratio de la durée de la transcription rapporté à la durée des corpus transcrits. Ce chantier est en cours et passe par l'utilisation de la capture de mouvement (Kinect pour le visage, centrales inertielles pour le reste du corps), couplant les données de chaque segment (main, avant-bras, bras, différentes parties du visage) avec des seuils établis lors de la phase de transcription manuelle actuelle. Une fois stabilisés, ces seuils devraient permettre de coupler directement les données de la capture de mouvement (Mocap) avec les caractères génériques de la police de caractère Typannot, sans passer par la phase de transcription manuelle. Conscients que ces stabilisations passent par des campagnes de transcription à venir, elles doivent finalement aboutir à une économie drastique de la durée de transcription, réduite alors à la seule segmentation. Le bénéfice devrait échoir également à toutes les manifestations multimodales de toute parole, y compris celle des langues vocales. 


\section{Références bibliographiques}

Abramova, E. (2018). The role of pantomime in gestural language evolution, its cognitive bases and an alternative. Journal of Language Evolution, 3(1), 26-40.

Bébian R.-A.-A. (1826). Mimographie, ou Essai d'écriture mimique propre à régulariser le langage des sourds-muets. Paris : L. Colas

http://archive.org/details/BebianBallyMimographieLangageSourdsMuets1826

Bellugi, U. \& Klima, E. S. (1979). The signs of language. Psychological Science, 1, 6-9.

Benesh, R. \& Benesh, J. (1956). An introduction to Benesh Dance Notation. London : A. \&C. Black.

Bianchini, C. S. (2012). Analyse métalinguistique de l'émergence d'un système d'écriture des Langues des Signes : SignWriting et son application à la Langue des Signes Italienne (LIS). Univ. Paris 8 (C. Cuxac \& D. Boutet) et Univ. Studi Perugia (M. Castelli, E. Antinoro Pizzuto): Paris.

Bianchini, C. S., Chèvrefils, L., Thomas, C., Danet, C., Doan, P., Rébulard, M., Contesse, A. \& Boutet, D. (2018). Guide d'annotation pour les polices de caractère Typannot dans le cadre du projet Typannot-Graphé. [rapport de recherche]. https://hal.archives-ouvertes.fr/hal-01965272

Bonnal-Vergès, F. (2005). Sémiogenèse de la langue des signes française : étude critique des signes de la langue des signes française attestés sur support papier depuis le XVIII siècle et nouvelles perspectives de dictionnaires. Université de Toulouse-Le Mirail, Toulouse.

Boutet, D. \& Blondel, M. (2016). Les corpus de Langue des Signes Française. in : La Langue des Signes Française (LSF), modélisations, ressources et applications. London : ISTE Editions, 47-85. https://halshs.archives-ouvertes.fr/halshs-01422734

Boutet, D. \& Garcia, B. (2007). Structuration morpho-phonétique de la Langue des Signes Française (LSF). Etude à partir d'une base de données. Atelier Traitement Automatique des Langues des Signes (TALS), TALN 2007. http://www.irit.fr/tals07/CP/Articles/Boutet.pdf.hal-00609047

Boutet, D., Sallandre, M.-A. \& Fusellier-Souza, I. (2010). Gestualité humaine et langues des signes : entre continuum et variations. Langage et société, 131(1), 55-74.

https://doi.org/10.3917/ls.131.0055

Brentari, D. (1998). A prosodic model of sign language phonology. Cambridge MS : The MIT Press.

Brown, P. \& Levinson, S. C. (1993). "Uphill" and "downhill" in Tzeltal. Journal of Linguistic Anthropology, 3(1), 46-74.

Chételat-Pelé, E. (2010). Les gestes non manuels en Langue des Signes Française, annotation, analyse et formalisation : application aux mouvements des sourcils et aux clignements des yeux (Université de Provence - Aix-Marseille I). http://tel.archives-ouvertes.fr/tel-00547777

Chevrefils, L. (à paraître). Relations entre les paramètres de l'emplacement et du mouvement en Langue des Signes Française. (titre prov.). Univ. Rouen Normandie (D. Boutet \& B. Guignard) : Rouen.

Darwin, C. (1998). The expression of the emotions in man and animals. Oxford University Press.

Dokic, J. \& Pacherie, E. (2006). On the very idea of frame of reference. in : Spaces in languages (M. Hickmann \& S. Robert, eds), Typological Studies in Language, 66. Amsterdam: John Benjamins, 259-280.

Eccarius, P. \& Brentari, D. (2008). Handshape coding made easier: a theoretically based notation for phonological transcription. Sign Language \& Linguistics, 11(1), 69-101.

Efron, D. (1972). Gesture, race and culture. The Hague : Mouton.

Emmorey, K. (2003). The neural systems underlying sign language. in : Oxford handbook of deaf studies, language, and education, 361-376.

Emmorey, K., McCullough, S., Mehta, S., Ponto, L. L. \& Grabowski, T. J. (2011). Sign language and pantomime production differentially engage frontal and parietal cortices. Language and Cognitive Processes, 26(7), 878-901. 
Feuillet, R. A. (1701). Chorégraphie ou l'art de décrire la dance, par caractères, figures et signes démonstratifs. Paris : Michel Brunet.

Goldin-Meadow, S. \& Brentari, D. (2017). Gesture, sign and language: the coming of age of sign language and gesture studies. Behavioral and Brain Sciences, e46, $82 \mathrm{p}$. doi:10.1017/S0140525X15001247

Hall, F. (1967). Benesh notation and ethnochoreology. Ethnomusicology, 11(2), 188-198.

Johnson, R. E. \& Liddell, S. K. (2010). Toward a phonetic representation of signs: sequentiality and contrast. Sign Language Studies, 11(2), 241-274. doi.org/10.1353/sls.2010.0008

Kühnl-Kinel, A. (1999). Marcel Marceau : contribution à l'étude de l'art du mime. Villeneuve d'Ascq : Presses Universitaires du Septentrion.

Lecoq, J., Carasso, J.-G. \& Lallias, J.-C. (1997). Le corps poétique : un enseignement de la création théâtrale. Arles : Actes Sud.

Levinson, S. C. (1996). Frames of reference and Molyneux's question: crosslinguistic evidence. in : Language, speech, and communication: language and space (P. Bloom, M. A. Peterson, L. Nadel \& M. F. Garrett eds). Cambridge MS : The MIT Press, 109-169.

Liddell, S. K. (2003). Grammar, gesture, and meaning in American Sign Language. Cambridge University Press.

McMonnies, C. W. (1999). Chart construction and letter legibility/readability. Ophthalmic and Physiological Optics, 19(6), 498-506. doi.org/10.1046/j.1475-1313.1999.00460.x

McNeill, D. (1992). Hand and mind: what gestures reveal about thought. Chicago IL : University of Chicago Press.

McNeill, D. (2005). Gesture and thought. Chicago IL : University of Chicago Press.

Napier, J. R. (1956). The prehensile movements of the human hand. Journal of Bone and Joint Surgery, 38(4), 902-913.

Pederson, E., Danziger, E., Wilkins, D., Levinson, S., Kita, S. \& Senft, G. (1998). Semantic typology and spatial conceptualization. Language, 74(3), 557-589.

Pezin, P. [éditeur] (2003). Étienne Decroux, mime corporel: Textes, études et témoignages. Saint-Jean de Védas : L'Entretemps Editions.

Prillwitz, S., Leven, R., Zienert, H., Hanke, T. \& Henning, J. (1989). Hamburg notation system for sign languages: an introductory guide. Hamburg : Signum Press.

Radutzky E. (2001). Dizionario elementare della Lingua dei Segni Italiana. Roma : Ed. Kappa.

Sandler, W. (1989). Phonological representation of the sign: linearity and nonlinearity in American Sign Language. Dordrecht : Walter de Gruyter.

Sandler, W. (2013). Vive la différence : sign language and spoken language in language evolution. Language and Cognition, 5(2-3), 189-203. doi.org/10.1515/langcog-2013-0013

Schembri, A., Jones, C. \& Burnham, D. (2005). Comparing action gestures and classifier verbs of motion: evidence from Australian Sign Language, Taiwan Sign Language, and nonsigners' gestures without speech. Journal of Deaf Studies and Deaf Education, 10(3), 272-290. doi.org/10.1093/deafed/eni029

Singleton, J. L., Goldin-Meadow, S. \& McNeill, D. (1995). The cataclysmic break between gesticulation and sign: evidence against a unified continuum of gestural communication. in : Language, gesture, and space (K. Emmorey \& J. Reilly eds). Hillsdale NJ : Lawrence Erlbaum Associates Publishers, 287-311.

Slobin, D. I., Hoiting, N., Anthony, M., Biederman, Y., Kuntze, M., Lindert, R., Pyers, J., Thumann, H. \& Weinberg, A. (2001). Sign language transcription at the level of meaning components: the Berkeley Transcription System (BTS). Sign Language \& Linguistics, 4(1-2), 63-104. doi.org/10.1075/sll.4.12.07slo 
Sloetjes, H. \& Wittenburg, P. (2008). Annotation by category: ELAN and ISO DCR. Proceedings $6^{\text {th }}$ International Conference on Languace Resources Evaluation (LREC 2008), 816-820.

Stokoe, W. C. (1960). Sign language structure: an outline of the visual communication systems of the American Deaf. Journal of Deaf Studies and Deaf Education, 10(1), 3-37. doi.org/10.1093/deafed/eni001

Stokoe, W. C. (1976). A dictionary of American Sign Language on linguistic principles. Silver Spring MD : Linstok Press.

Sutton, V. (1972). Sutton Movement Writing. La Jolla CA : Center for Sutton Movement Writing.

Sutton, V. (1995). Lessons in SignWriting. La Jolla CA : Deaf Action Committee for SignWriting.

Tabourot, J. (1588). Orchesographie et traicté en forme de dialogue, par lequel toutes personnes peuvent facilement apprendre \& practiquer l'honneste exercice des dances. Lengres: Ichan des Preyz Imprimeur.

Talmy, L. (1983). How language structures space. in : Spatial orientation: theory, research and application (H. Pick ed.). New York NY : Springer, 225-282.

Thomas, C. (à paraître). Le rôle des articulateurs non-manuels en langues des signes française (titre prov.). Univ. Rouen Normandie (D. Boutet) : Rouen.

van der Hulst, H. \& Channon, R. (2010). Notation systems. in : Sign Languages (D. Brentari ed.). Cambridge University Press, 151-172.

http://homepage.uconn.edu/ hdv02001/Articles-pdfs/131\%20-\%20Notation\%20Systems.pdf

von Laban, R. \& Lange, R. (1975). Laban's principles of dance and movement notation. Boston MS : Princeton Book Co Pub.

${ }^{1}$ L'équipe GestualScript est constituée de linguistes, de designers de caractères et d'informaticiens. En 2020, elle a perdu l'un de ses membres fondateurs, Dominique Boutet (1966-2020), dont les travaux sur une approche kinésiologique de la gestualité ont été fondamentaux pour le développement de Typannot, et dont l'enthousiasme et le plaisir de la découverte vont continuer à guider l'équipe GestualScript.

${ }^{2}$ Fonctionnel versus formel; extensivité vs spécificité du système; niveau de formalisation; prise en compte de l'iconicité des signes et gestes, motivation entre les signes et les symboles graphiques utilisés; lien avec la LV dominante et son système graphique; etc. (pour des typologies : van der Hulst \& Channon [2010]).

${ }^{3}$ Configuration $=$ conformation stable au cours du signe des doigts et de la main; emplacement $=$ localisation de chacune des mains; orientation $=$ direction de chaque paume; mouvement $=$ déplacement des mains dans l'espace.

${ }^{4}$ Stokoe Notation a connu, au cours des années, de nombreuses adaptations qui ont permis d'en affiner le potentiel descriptif sans en altérer la nature. Il est proposé ici d'utiliser la version de Stokoe Notation développée par E. Radutzky (2001).

${ }^{5}$ Cette séquence se lit : la main dominante en configuration dite 5 , les doigts dressés vers le haut, la paume orientée vers l'intérieur $(\Lambda>)$, est située aux environs de la main non dominante (ND) et fait un mouvement de torsion répété $(\omega \cdot)$; l'autre main, dominée, en configuration $B$, a la paume orientée vers le bas $(<\Lambda)$, elle ne bouge pas et est en contact $(*)$ avec le coude du segment dominant (gd).

${ }^{6}$ Sur le membre supérieur deux DDL ont leur axe qui court le long d'un os voire entre deux os (la RINT/REXT du bras et la PRONO-SUPI de l'avant-bras).

${ }^{7}$ Appelés parts, variables et values dans nos articles en anglais.

${ }^{8}$ Appelés genericity, legibility, scriptability et modularity dans nos articles en anglais.

${ }^{9}$ Typannot est notamment utilisé dans deux thèses en cours, traitant le mouvement des segments du membre supérieur (Chevrefils [à paraitre]) et les paramètres non-manuels (Thomas [à paraitre]).

${ }^{10} \mathrm{https}: / /$ cocoon.huma-num.fr/exist/crdo/meta/cocoon-30180d73-59a4-316f-aff8-a9a0399e666a

Les nouvelles transcriptions sont en cours de dépôt sur la plateforme Cocoon. 